\title{
Limits on decaying dark energy density models from the CMB temperature-redshift relation
}

\author{
Jetzer, P ; Puy, D ; Signore, M ; Tortora, C
}

\begin{abstract}
The nature of the dark energy is still a mystery and several models have been proposed to explain it. Here we consider a phenomenological model for dark energy decay into photons and particles as proposed by Lima (Phys Rev D 54:2571, 1996). He studied the thermodynamic aspects of decaying dark energy models in particular in the case of a continuous photon creation and/or disruption. Following his approach, we derive a temperature redshift relation for the cosmic microwave background (CMB) which depends on the effective equation of state w eff and on the "adiabatic index" . Comparing our relation with the data on the CMB temperature as a function of the redshift obtained from Sunyaev-Zel'dovich observations and at higher redshift from quasar absorption line spectra, we find $\mathrm{w}$ eff $=-0.97 \pm 0.03$, adopting for the adiabatic index $=4 / 3$, in good agreement with current estimates and still compatible with $\mathrm{w}$ eff $=-1$, implying that the dark energy content being constant in time.
\end{abstract}

DOI: https://doi.org/10.1007/s10714-010-1091-4

Posted at the Zurich Open Repository and Archive, University of Zurich

ZORA URL: https://doi.org/10.5167/uzh-48325

Journal Article

Accepted Version

Originally published at:

Jetzer, P; Puy, D; Signore, M; Tortora, C (2011). Limits on decaying dark energy density models from the CMB temperature-redshift relation. General Relativity and Gravitation, 43(4):1083-1093.

DOI: https://doi.org/10.1007/s10714-010-1091-4 


\title{
Limits on decaying dark energy density models from the CMB temperature-redshift relation
}

\author{
Philippe Jetzer ${ }^{1,2},{ }^{*}$ Denis Puy ${ }^{2},{ }^{\dagger}$ Monique Signore ${ }^{3},{ }^{\ddagger}$ and Crescenzo Tortora ${ }^{1 \S}$ \\ (1) Institut für Theoretische Physik, Universität Zürich, \\ Winterthurerstrasse 190, CH-8057 Zürich, Switzerland \\ (2) Groupe de Recherche d'Astronomie et d'Astrophysique du Languedoc, \\ Université des Sciences Montpellier II, GRAAL CC72, F-34095 Montpellier cedex 09, France and \\ (3) Observatoire de Paris, LERMA, 61 av. de l'Observatoire, F-75014 Paris, France
}

\begin{abstract}
The nature of the dark energy is still a mystery and several models have been proposed to explain it. Here we consider a phenomenological model for dark energy decay into photons and particles as proposed by Lima [1]. He studied the thermodynamic aspects of decaying dark energy models in particular in the case of a continuous photon creation and/or disruption. Following his approach, we derive a temperature redshift relation for the CMB which depends on the effective equation of state $w_{\text {eff }}$ and on the "adiabatic index" $\gamma$. Comparing our relation with the data on the CMB temperature as a function of the redshift obtained from Sunyaev-Zel'dovich observations and at higher redshift from quasar absorption line spectra, we find $w_{\text {eff }}=-0.97 \pm 0.034$, adopting for the adiabatic index $\gamma=4 / 3$, in good agreement with current estimates and still compatible with $w_{\text {eff }}=-1$, implying that the dark energy content being constant in time.
\end{abstract}

PACS numbers:

Keywords:

\section{INTRODUCTION}

After the discovery that the cosmic expansion is accelerating $[2,3]$ and the first cosmic microwave background radiation $(\mathrm{CMB})$ observations of a flat universe [4], the current standard model of cosmology implies the existence of dark energy which accounts for about $70 \%$ of the total energetic content of the universe, which according to the observations is spatially flat [5]. The nature of the dark energy is still a mystery (see for instance the review of [6]).

Several models have been proposed to explain dark energy [7-15]. An alternative consists to consider a phenomenological decaying dark energy density with continuous creation of photons [16, 18] or matter [15]. The dark energy might decay slowly in the course of the cosmic evolution and thus provide the source term for matter and radiation. Different such models have been discussed and strong constraints come from very accurate measurements of the CMB.

$\mathrm{CMB}$ is the best evidence for an expanding Universe starting from an initial high density state. Within the Friedmann-Robertson-Walker (FRW) models of the Universe the radiation after decoupling expands adiabatically and scales as $(1+z), z$ being the redshift. Depending on the decay mechanism of the dark energy the created photons could lead to distortions in the Planck spectrum of the CMB. If the dark energy is considered as a second fluid component transferring energy continuously to the material component, the second law of thermodynamics constrains the whole process in such a way that the temperature law, that is how the temperature of the CMB changes with time, can be determined. Such a model has been discussed in detail by Lima [1], who also established under which conditions the equilibrium relations are preserved. He also introduced the concept of an "adiabatic" vacuum decay. In a further paper Lima et al. [16] following the approach outlined in [1] derived a temperature redshift relation law $T=(1+z)^{1-\beta}$ assuming a nonvanishing source term in the balance equation for particle number, which depends on a function $\beta$ assumed to be constant. This parameter can then be determined by fitting the temperature redshift relation to the data. However, being a purely phenomenological parameter it cannot be related to more fundamental quantities such as for instance the effective equation of state of the dark fluid $w_{\text {eff }}$.

In this paper we follow the same approach by Lima [1], we do not restrict to the case of a universe dominated by a radiation fluid as in [16], since we leave the adiabatic index $\gamma$ (which appears in the equation of state of the fluid including both matter and radiation) free to vary and in particular we do not adopt their simplifying assumption

\footnotetext{
*Electronic address: jetzer@physik.uzh.ch

${ }^{\dagger}$ Electronic address: puy@graal.univ-montp2.fr

‡Electronic address: monique.signore@obspm.fr

$\S$ Electronic address: ctortora@physik.uzh.ch
} 
of a constant $\beta$ and thus we derive a temperature redshift relation for CMB which depends on more fundamental quantities such as $w_{\text {eff }}$ and $\gamma$. We compare our temperature redshift relation with the available data on the CMB temperature as a function of the redshift obtained from Sunyaev-Zel'dovich observations and at higher redshift from quasar absorption line spectra.

The paper is organized as follows. In $\S$ II we will introduce the theoretical background for our model, deriving the main quantities of interest. In $\S$ III the multi-redshift measurements of the CMB temperature are presented, while $\S \mathrm{IV}$ and $\S \mathrm{V}$ are devoted to the discussion of the results, conclusions and future prospects.

\section{TEMPERATURE-REDSHIFT RELATION}

We assume the usual Friedmann-Robertson-Walker (FRW) cosmology and that the material medium of the Universe contains three different components: a fluid, which includes radiation and matter (both baryonic and dark matter) as particular cases, for which we have the law

$$
p=(\gamma-1) \rho
$$

with $\gamma$ in the interval $[0-2]$. Further we assume a dark energy, quintessence-like $x$ component, with pressure $p_{x}$ and density $\rho_{x}$ and a 'bare' cosmological constant $\Lambda_{0}$. With these components we get for the Einstein field equations

$$
\begin{gathered}
8 \pi G\left(\rho+\rho_{x}\right)+\Lambda_{0}=3 \frac{\dot{R}^{2}}{R^{2}}+3 \frac{k}{R^{2}}, \\
8 \pi G\left(p+p_{x}\right)-\Lambda_{0}=-2 \frac{\ddot{R}}{R}-\frac{\dot{R}^{2}}{R^{2}}-\frac{k}{R^{2}},
\end{gathered}
$$

where a dot means time derivative. For the dark energy we assume the relation $p_{x}=-\rho_{x}$ (thus setting $w_{x}=-1$ ) to hold. This is for instance the case for quintessence models in the limit where the scalar field does not depend on time and thus its time derivative vanishes. In the later stages of the Universe the time dependence is possibly very weak so that $w_{x}=-1$ holds up to small corrections, which we will neglect in the following, thus simplifying our calculations. This can be viewed as a first order approximation.

Furthermore, we assume that there is no curvature, thus $k=0$, and take the sum of eqs. (2) and (3). This way we get

$$
8 \pi G(\rho+p)=2 \frac{\dot{R}^{2}}{R^{2}}-2 \frac{\ddot{R}}{R}=-2 \dot{H} .
$$

Following the paper by Lima et al. [16] the energy conservation equation can be written as

$$
\dot{\rho}+3(\rho+p) H=C_{x}
$$

where $H=\dot{R} / R$ is the Hubble parameter and

$$
C_{x}=-\dot{\rho}_{x}-3\left(\rho_{x}+p_{x}\right) H
$$

is a term which depends on the dark fluid and acts as a source term for the $\gamma$-fluid energy. Evidently, if no interaction between the different fluids exists, then $C_{x}$ is null and the standard picture is recovered. $C_{x}$ can describe different physical situations such as for instance a thermogravitational quantum creation theory [17] or a quintessence scalar field cosmology [13].

Assuming as mentioned above the relation $p_{x}=-\rho_{x}$ and writing $\rho_{x}=\Lambda(t) /(8 \pi G)$, we have

$$
C_{x}=-\frac{\dot{\Lambda}(t)}{8 \pi G}
$$

On the other hand the equation for the particle number density is given by 


$$
\dot{n}+3 n H=\psi,
$$

where $n$ is the particle number density and $\psi$ is the particle source term. Using Gibbs law and well-known thermodynamic identities, following the derivation given in the paper by Lima et al. [16], one gets

$$
\frac{\dot{T}}{T}=\left(\frac{\partial p}{\partial \rho}\right)_{n} \frac{\dot{n}}{n}-\frac{\psi}{n T\left(\frac{\partial \rho}{\partial T}\right)_{n}}\left[p+\rho-\frac{n C_{x}}{\psi}\right] .
$$

To get a black-body spectrum the second term in brackets in eq.(9) has to vanish, thus

$$
C_{x}=\frac{\psi}{n}[p+\rho]
$$

Note that $C_{x}$ is null if $p=-\rho$, (this is the trivial case when only a dark fluid exists) or if $\psi=0$, which means that both the dark and normal fluids are separately conserved. This way we get with eq.(1)

$$
C_{x}=\frac{\psi}{n}[(\gamma-1) \rho+\rho]=\frac{\psi \gamma \rho}{n}
$$

and eq.(9) becomes

$$
\frac{\dot{T}}{T}=\left(\frac{\partial p}{\partial \rho}\right)_{n} \frac{\dot{n}}{n}
$$

With $\left(\frac{\partial p}{\partial \rho}\right)_{n}=(\gamma-1)$ one obtains

$$
\frac{\dot{T}}{T}=(\gamma-1) \frac{\dot{n}}{n} .
$$

Using the equation for the particle number conservation eq.(8) into eq.(13) leads to

$$
\frac{\dot{T}}{T}=(\gamma-1)\left[\frac{\psi}{n}-3 H\right]
$$

With eqs.(10) and (7) we get

$$
\frac{\dot{T}}{T}=(\gamma-1)\left[-\frac{\dot{\Lambda}}{8 \pi G(p+\rho)}-3 H\right] .
$$

We insert (4) in eq. (15) and obtain

$$
\frac{\dot{T}}{T}=(\gamma-1)\left[\frac{\dot{\Lambda}}{2 \dot{H}}-3 H\right] .
$$

As next we integrate eq.(16)

$$
\int_{t_{1}}^{t_{0}} \frac{\dot{T}}{T} d t=(\gamma-1) \int_{t_{1}}^{t_{0}}\left[\frac{\dot{\Lambda}}{2 \dot{H}}-3 H\right] d t,
$$

where $t_{0}$ denotes the present time and $t_{1}$ some far instant in the past. With $H=\dot{R} / R$ the second term in the bracket on the right and the term on left hand side can be immediately integrated. Indeed, if $\dot{\Lambda}$ vanishes and $\gamma=4 / 3$ one 
gets the usual dependence $T(t)=\frac{R\left(t_{1}\right) T\left(t_{1}\right)}{R(t)}$ for a radiation fluid. Whereas to carry out the integration of the first term on the right hand side it is useful to perform a change of variable from $t$ to $z$ and accordingly $\frac{d t}{d z}=\frac{-1}{H(1+z)}$. This way we get (with $z_{1}$ corresponding to the time $t_{1}$ and $z_{0}=0$ corresponding to $t_{0}$ present time)

$$
\ln \frac{T(z=0)}{T\left(z_{1}\right)}+3(\gamma-1) \ln \frac{R(z=0)}{R\left(z_{1}\right)}=\frac{(\gamma-1)}{2} \int_{0}^{z_{1}} \frac{\Lambda^{\prime}}{H^{\prime} H(1+z)} d z,
$$

where' denotes derivative with respect to $z$. We now assume a power law model for the $\Lambda$ term, thus $\Lambda=B\left(R / R_{0}\right)^{-m}$ or $\Lambda=B(1+z)^{m}$, where $B$ is a constant, which is $B=3 H_{0}^{2}\left(1-\Omega_{m 0}\right)$ [15], if the "bare" cosmological constant $\Lambda_{0}$ vanishes.

For such a model the Hubble parameter as a function of $m$ can be computed [15] and leads to

$$
H(z)=H_{0}\left[\frac{\left(3 \Omega_{m 0}-m\right)}{3-m}(1+z)^{3}+\frac{3\left(1-\Omega_{m 0}\right)}{3-m}(1+z)^{m}\right]^{1 / 2},
$$

where $\Omega_{m 0}$ is related to the present value of the total matter density: $\rho_{m 0}=3 H_{0}^{2} M_{P l}^{2} \Omega_{m 0}$, with $M_{P l}=(8 \pi G)^{-1 / 2}$. Due to the negligible energy density accounted for by the radiation, this term has been neglected here. As next we insert $H(z)$ and its derivative as taken from eq.(19) into eq.(18) and integrate it, to get (setting $z_{1}=z$ )

$$
T(z)=T_{0}\left(\frac{R_{0}}{R(z)}\right)^{3(\gamma-1)} \exp \left(\frac{B(1-\gamma)}{3 H_{0}^{2}\left(\Omega_{m 0}-1\right)} A\right)
$$

where

$$
A=\left[\ln \left(\left(m-3 \Omega_{m 0}\right)+m(1+z)^{m-3}\left(\Omega_{m 0}-1\right)\right)-\ln \left((m-3) \Omega_{m 0}\right)\right] .
$$

We can also write eq. (20) as

$$
T(z)=T_{0}(1+z)^{3(\gamma-1)}\left(\frac{\left(m-3 \Omega_{m 0}\right)+m(1+z)^{m-3}\left(\Omega_{m 0}-1\right)}{(m-3) \Omega_{m 0}}\right)^{\gamma-1} .
$$

We inserted in the exponent of eq.(20) the explicit form of $B$, thus getting as exponent in the above eq. $(\gamma-1)$. Notice that for $z=0$ : $T(0)=T_{0}$, whereas for $m=0$ the expression in the parenthesis is equal to 1 and thus $T(z)=T_{0}(1+z)^{3(\gamma-1)}$, which for the canonical value of $\gamma=4 / 3$ reduces to the standard expression. Also for $m=3$, although not a realistic value, the expression in the parenthesis is non-singular and equal to: $1+\frac{3 \ln (1+z)\left(\Omega_{m 0}-1\right)}{\Omega_{m 0}}$.

Since from the analysis by Ma [15] we expect $m$ to be small we give here an expression for $T(z)$, where we keep only the leading linear terms in the expansion in $m$ and neglect all terms of higher order. This way we get from eq. $(22)$

$$
T(z)=T_{0}(1+z)^{3(\gamma-1)}\left(1+\frac{m}{3}-\frac{m}{3 \Omega_{m 0}}\left(1+(1+z)^{-3}\left(\Omega_{m 0}-1\right)\right)\right)^{(\gamma-1)} .
$$

If $m$ is positive, then the dark energy slowly decreases as a function of the cosmic time, whereas if $m$ is negative the inverse process happens.

It is easy to calculate the equivalent effective dark energy equation of state $p=w_{\text {eff }} \rho$ with $w_{\text {eff }}=\frac{m}{3}-1$. If $m>0$ then we have $w_{\text {eff }}>-1$, i.e. our model is quintessence-like $[9,12,13]$, while we have a phantom-like [11] model when $m$ is negative and $w_{e f f}<-1$. Another interesting quantity is the deceleration parameter, which can be written as

$$
q(z)=-\frac{\ddot{R} R}{\dot{R}^{2}}=\frac{(1+z)^{3}\left(m-3 \Omega_{m 0}\right)+3(m-2)(1+z)^{m}\left(\Omega_{m 0}-1\right)}{2(1+z)^{3}\left(m-3 \Omega_{m 0}\right)+6(1+z)^{m}\left(\Omega_{m 0}-1\right)} .
$$

Imposing that $q(z)=0$, we can determine the transition redshift, i.e. the redshift when the Universe was changing from a deceleration to an acceleration phase, which is given by

$$
z_{T}=\left(\frac{3(2-m)\left(1-\Omega_{m 0}\right)}{3 \Omega_{m 0}-m}\right)^{\frac{1}{3-m}}-1 .
$$

From this result we have that the larger $m$ is, the earlier the Universe changes from deceleration to acceleration. 


\section{MULTI-REDSHIFT MEASUREMENTS OF $T_{\mathrm{CMB}}$ AND FITTING PROCEDURE}

To test the decaying $\Lambda$ model we will rely on the CMB temperatures derived from the absorption lines of high redshift systems and the ones from Sunyaev-Zel'dovich effect in clusters of galaxies (we will collectively quote as $T_{C M B}$, hereafter).

We can infer the CMB temperature at high redshift from the analysis of quasar absorption line spectra which give atomic or ionic fine structure levels excited by the photo-absorption of the cosmic microwave background radiation. Detection of absorption from the ground states and excited of $C_{I}$ in damped Ly $\alpha$ system towards quasars permits to estimate the population ratio of the excited fine-structure levels, and thus to derive the CMB temperature at the redshift of the damped Ly $\alpha$ system. Ge et al. [19], in the damped Ly $\alpha$ system of the QSO 0013-004, found the CMB temperature

$$
T_{\mathrm{CMB}}=7.9 \pm 1.0 \mathrm{~K} \text { at } z=1.9731 .
$$

Srianand et al. [20] detected absorption lines in an isolated gas cloud toward PKS1232+0815, with which they could get the following limits on the CMB temperature

$$
6.0<T_{\mathrm{CMB}}<14 \mathrm{~K} \text { at } z=2.33771,
$$

and Molaro et al. [21] in the damped Ly $\alpha$ system toward QSO 0347-3819 gave

$$
T_{\mathrm{CMB}}=12.1_{-3.2}^{+1.7} \mathrm{~K} \text { at } z=3.025 .
$$

The cosmic microwave background can also excite levels of molecular species, when the energy separation involved corresponds to the CMB peak frequency. We know that molecular gas is an important ingredient of star formation. Damped Ly $\alpha$ absorbers (i.e. DLAs) are generally taken as seeds of present-day galaxies, and for this reason DLAs can be considered as an important gas reservoir for star formation. $\mathrm{H}_{2}$, the most abundant molecules in the Universe is a good candidate (see Puy et al. [22], Galli \& Palla [23] and Stancil et al. [24]). The search of $\mathrm{H}_{2}$ in DLAs can be carried out by observing the $\mathrm{H}_{2}$ absorption lines in the lyman $\mathrm{X}^{1} \Sigma_{\mathrm{g}}^{+} \longmapsto \mathrm{B}^{1} \Sigma_{\mathrm{u}}^{+}$. Cui et al. [25] obtained the spectrum of molecular hydrogen associated with the damped Ly $\alpha$ system at $z=1.7765$ toward the quasar Q1331+170 and constructed a model to describe the structure of $\mathrm{H}_{2}$ absorber. Applying the inferred conditions to the $\mathrm{C}_{\mathrm{I}}$ fine structure excitation, they found the CMB temperature to be

$$
T_{\mathrm{CMB}}=7.2 \pm 0.8 \mathrm{~K} \text { at } z=1.7765 .
$$

Srianand et al. [26], from the CO rotational excitation temperatures, derive in a damped Ly $\alpha$ system towards SDSS $\mathrm{J} 143912.04+111740.5$

$$
T_{\mathrm{CMB}}=9.15 \pm 0.72 \mathrm{~K} \text { at } z=2.41837 \text {. }
$$

During passage through a cluster of galaxies some of the photons of the cosmic microwave background radiation are scattered by electrons in the hot intracluster medium. This imprint was first described by Sunyaev-Zel'dovich [27]. Thus, spectral measurements of galaxy clusters at different frequency bands yield independent intensity ratios for each cluster. The combinations of these measured ratios permit to extract the cosmic microwave background radiation (see Fabbri et al. [28]). Recently, Luzzi et al. [29] have analyzed the results of multifrequency Sunyaev-Zel'dovich measurements toward several clusters from 5 telescopes (BIMA, OVRO, SUZI II, SCUBA and MITO), see Table 1.

We will match the observed $T_{C M B}$ with the theoretical expression $T_{t h}$, which we have derived in eq. (22), by minimizing the following merit function

$$
\chi_{T C M B}^{2}=\sum_{i=1}^{N_{T C M B}}\left(\frac{T_{t h}^{i}-T_{C M B}^{i}}{\sigma_{C M B, i}}\right)^{2},
$$

where $\sigma_{C M B, i}$ is the error on the temperature estimates and $N_{T C M B}=18$ is the number of available observational data. We will find the best fitted parameters which correspond to the minimum of $\chi^{2}$, i.e. $\chi_{\min }^{2}$ and we will determine the $68 \%$ uncertainties by imposing $\Delta \chi^{2}=\chi^{2}-\chi_{\min }^{2}=1$. 
TABLE I: CMB $T_{\mathrm{CMB}}$ values for several clusters from Luzzi et al. [29].

\begin{tabular}{lcc}
\hline \hline Cluster & $\begin{array}{c}T_{\mathrm{CMB}} \\
(\mathrm{K})\end{array}$ & $z$ \\
\hline A1656 & $2.72 \pm 0.10$ & 0.023 \\
A2204 & $2.90 \pm 0.17$ & 0.152 \\
A1689 & $2.95 \pm 0.27$ & 0.183 \\
A520 & $2.74 \pm 0.28$ & 0.200 \\
A2163 & $3.36 \pm 0.20$ & 0.202 \\
A773 & $3.85 \pm 0.64$ & 0.216 \\
A2390 & $3.51 \pm 0.25$ & 0.232 \\
A1835 & $3.39 \pm 0.26$ & 0.252 \\
A697 & $3.22 \pm 0.26$ & 0.282 \\
ZW3146 & $4.05 \pm 0.66$ & 0.291 \\
RXJ1347 & $3.97 \pm 0.19$ & 0.451 \\
CL0016+16 & $3.69 \pm 0.37$ & 0.546 \\
MS0451 & $4.59 \pm 0.36$ & 0.550 \\
\hline
\end{tabular}

\section{RESULTS}

We have tested our model by comparing the CMB temperature predicted (see eq. 22), with the collection of multiredshift measurements of $T_{\mathrm{CMB}}$ we have discussed in the previous section. We set $T_{0}=2.725 \mathrm{~K}$, which is quite well determined in the literature [30], and the matter density $\Omega_{\mathrm{m} 0}=0.273$ to the value inferred in Komatsu et al. [31].

If we take $\gamma=4 / 3$, then we find $m=0.09 \pm 0.10$ and $z_{T}=0.82 \pm 0.10$, while, if we leave both $\gamma$ and $m$ free to vary $m=0.20_{-0.23}^{+0.23}$ and $\gamma=1.35_{-0.05}^{+0.03}$ and $z_{T}=1.02 \pm 0.23$. In both the cases, the best fitted $m$ values are positive, but consistent with 0 within $1 \sigma$ uncertainty, while in the latter case the estimated value for $\gamma$ of 1.35 is consistent within the errors with the canonical value for radiation. Within the uncertainties, the derived $z_{T}$ are consistent with the typical values in literature (e.g. [10,32]), although, on average, higher. In Fig. 1 we plot the $T-z$ diagram with the different best fit curves.

Assuming a constant value for the ratio $\Psi / 3 n H=\beta$, with the constraint $0 \leq \beta \leq 1$, Lima et al. [16] found as a result instead of our eq.(22) the relation

$$
T(z)=T_{0}(1+z)^{1-\beta} .
$$

For a value of $\beta$ different from zero the temperature of the expanding universe at high values of $z$ is slightly lower than in the standard photon-conserved scenario. Lima et al. [16] discussed the various upper limits which can be derived on $T(z)$. The best values are provided by absorption lines from molecules. The results are presented in Fig. 1 of their paper, from which one can see that the upper limits are well compatible with the standard photon-conserved scenario, i.e. $\beta=0$. Luzzi et al. [29] found using their data (as given in our Table 1) and also the values mentioned in eqs. (23)-(26) as best fit the value $\beta=0.024_{-0.024}^{+0.068}$. We performed, as a check, the same fit using the same data with in addition the value given in eq.(27) and got $\beta=0.027_{-0.027}^{+0.04}$, which clearly is consistent with their value. Fitting several data sets to constrain the $H(z)$ function (as given by eq. 19), Ma [15] obtained $m=-0.09_{-0.11}^{+0.08}$ and $\Omega_{m 0}=0.29_{-0.07}^{+0.03}$. This model with negative $m$ is plotted as well in Fig. 1 as a comparison, although it is ruled out by our fitting of the CMB.

\section{CONCLUSIONS}

We studied a model for the dark energy decay based on the assumption that the dark energy is regarded as a second fluid component transferring energy continuously to the material component as suggested by Lima [1]. In which case the second law of thermodynamics constrains the whole process, thus allowing to determine the temperature redshift relation. As a new point we were able to derive for the latter quantity a relation depending on parameters such as $w_{\text {eff }}$ and $\gamma$. Thanks to recent CMB temperature measurements via the Sunyaev-Zel'dovich effect and others at higher redshift from quasar absorption lines, we could determine best fit values for these parameters. In particular, assuming the canonical $4 / 3$ value for $\gamma$ we found $m=0.09 \pm 0.10$ corresponding to $w_{\text {eff }}=-0.97 \pm 0.034$, whereas leaving $\gamma$ 


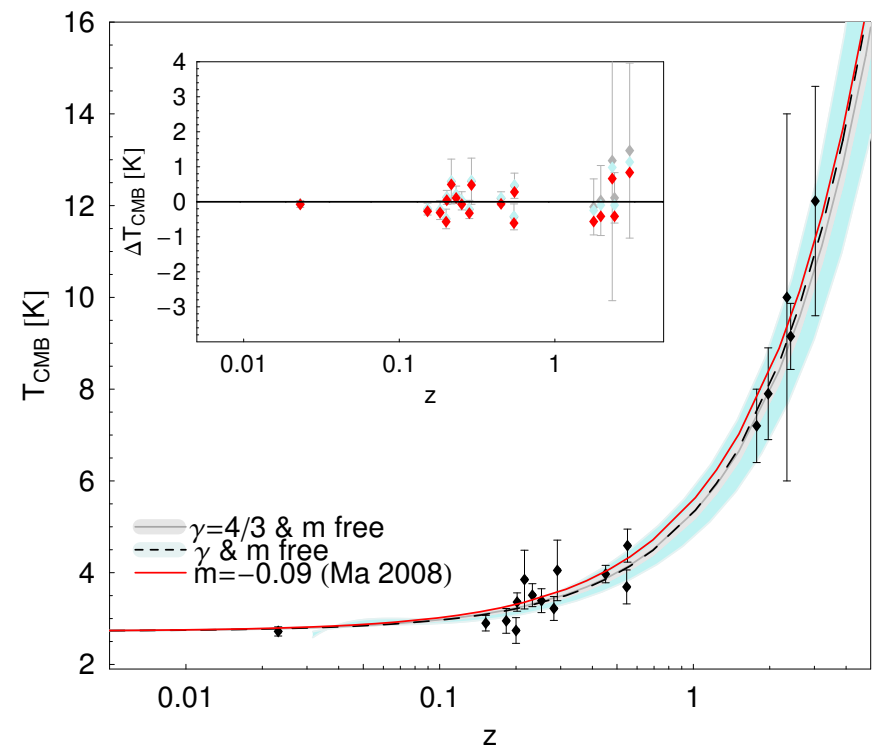

FIG. 1: Cosmic microwave background temperature for different m-models. The data are plotted as black symbols. As shown in the legend, the gray line and the lighter gray region are the best fitted curve and $1 \sigma$ scatter for the case with $\gamma=4 / 3$ and $m$ free. The black dashed line with the cyan region are relative to the case with both $\gamma$ and $m$ free. Finally the red curve is the best fit curve when using the value for $\mathrm{m}$ as found by Ma [15]. In the inset, we show the difference between the data points and each best fitted curve as a function of redshift.

open we got $m=0.20 \pm 0.10$ or $w_{\text {eff }}=-0.93 \pm 0.08$. Both results are within $1 \sigma$ consistent with the canonical value of -1 for the cosmological constant. Using SNe Ia data combined with CMB and Baryon Acoustic Oscillations, and assuming a flat universe, Kowalski et al. [33] found $w_{\text {eff }}=-0.97 \pm 0.06 \pm 0.06$ (stat, sys) and similarly Kessler et al. [34] estimated $w_{\text {eff }}=-0.96 \pm 0.06 \pm 0.12$. Our best fit value for $w_{\text {eff }}$ compares quite well with these values, and we point out that our "derivation" is completely different and to our knowledge new. The values $z_{T}$ we find for the transition redshift are in good agreement within the uncertainties with the typical ones quoted in the literature.

Clearly, it would be nice to have better measurements for the CMB temperature at high redshift, since the difference between the different models increases for high $z$ values. We notice also that since the best fit value for $m$ is small, it implies that the dark energy variation with time is very slow (at least when not considering too high $z$ values, where we expect our approximations to break down) and thus our assumption on $w_{x}=-1$ can be considered as justified a posteriori.

Acknowledgements

The authors thank Y. Chastagnier and Profs M. De Petris, Y. Rephaeli and N. Straumann for useful discussions. $\mathrm{Ph}$. Jetzer, thanks the French Regional Council of Languedoc Roussillon and the Physics Institute of Montpellier (IPM) for the invitation at the Laboratory of Astrophysics of the University of Montpellier. M. Signore thanks the Programme National de Cosmologie et Galaxie (PNCG) and the Swiss National Science Foundation for her many visits at the University of Montpellier and the University of Zurich. C. Tortora was supported by the Swiss National Science Foundation.

[1] J.A.S. Lima, Phys. Rev. D. 54, 2571 (1996)

[2] S. Perlmutter et al., Astrophys.J. 517, 565 (1999)

[3] A.G.Reiss et al., Astron.J. 116, 1009 (1998)

[4] P.de Bernardis et al., Nature 404, 955 (2000)

[5] D.N. Spergel et al., Astrophys. J. Suppl. 170, 377 (2007)

[6] R.Caldwell \& M.Kamionkowski, Ann.Rev.Nucl.Part.Sci. 59, 397 (2009)

[7] P. J. E. Peebles and B. Rathra, Rev. Mod. Phys. 75, 559 (2003)

[8] T. Padmanabhan, Phys. Rep. 380, 235 (2003)

[9] M. Demianski, E. Piedipalumbo, C. Rubano, C. Tortora, A\&A 431 27D (2005)

[10] V. F. Cardone, C. Tortora, A. Troisi, and S. Capozziello, Phys. Rev. D73, 043508 (2006) 
[11] R.R. Caldwell, Phys. Lett. B 545, 23 (2002)

[12] P.J.E. Peebles \& B. Ratra, Astrophys. J. Lett. 325, L17 (1988)

[13] B. Ratra \& P.J.E. Peebles, Phys. Rev. D 37, 3406 (1988)

[14] V. Sahni \& A.A. Starobinsky, Int. J. Mod. Phys. D9 373 (2000)

[15] Y. Ma, Nucl. Phys. B 804, 262 (2008)

[16] J.A.S. Lima, A.I. Silva and S.M. Viegas, Mon. Not. R. Astron. Soc. 312, 747 (2000)

[17] J.A.S. Lima and J.S. Alcaniz, Astron. and Astrophys. 348, 1 (1999)

[18] D. Puy, Astron. and Astrophys. 422, 1 (2004)

[19] J. Ge, J. Bechtold and J. Black, Astrophys. J 474, 67 (1997)

[20] R. Srianand, P. Petijean and C. Ledoux, Nature 408, 931 (2000)

[21] P. Molaro, S. Levshakov, M. Dessauges-Zavadsky and S. D'Odorico, Astron. and Astrophys. 381, L64 (2002)

[22] D. Puy, G. Alecian, J. Leorat, J. Lebourlot and G. Pineau des Forets, Astron. and Astrophys. 267, 337 (1993)

[23] D. Galli and F. Palla, Astron. and Astrophys. 335, 403 (1998)

[24] P. Stancil, S. Lepp and A. Dalgarno, Astrophys. J. 509, 1 (1998)

[25] J. Cui, J. Bechtold, J. Ge and D. Meyer, Astrophys. J. 633, 649 (2005)

[26] R. Srianand, P. Noterdaeme, C. Ledoux and P. Petijean, Astron. and Astrophys. 482, L39 (2008)

[27] R. Sunyaev and Y. Zel'dovich, Comm. Ap. Sp. Phys.4, 173 (1972)

[28] R. Fabbri, F. Melchiorri and V. Natale, Astrophys. Sp. Sci. 59, 223 (1978)

[29] G. Luzzi, M. Shimon, L. Lamagna, Y. Rephaeli, M. De Petris, A. Conte, S. De Gregori and E. Battistelli, Astrophys. J. 705, $1122(2009)$

[30] J.C. Mather et al., Astrophys. J. 512, 511 (1999)

[31] E. Komatsu et al., Astrophys. J. Suppl. 180, 330 (2009)

[32] R.A. Daly, et al., Astrophys. J. 677, 1 (2008)

[33] M. Kowalski et al. Astrophys. J. 686, 749 (2008)

[34] R. Kessler et al. Astrophys. J. Supp. 185, 32 (2009) 